\title{
STABILIZATION OF THE UNICYCLE VIA DYNAMIC FEEDBACK LINEARIZATION
}

\author{
Alessandro De Luca \\ Giuseppe Oriolo \\ Dipartimento di Informatica e Sistemistica, Università degli Studi \\ di Roma "La Sapienza", Via Eudossiana 18, 00184 Roma, Italy \\ \{deluca, oriolo, venditt\}olabrob.ing.uniroma1.it
}

Marilena Vendittelli

\begin{abstract}
We consider the feedback control problem for a wheeled mobile robot with the kinematics of a unicycle, a typical example of nonholonomic robotic system. It is shown that dynamic feedback linearization can be used to design a simple control law which is valid for trajectory tracking as well as point stabilization tasks. In particular, for both cases exponential convergence with linear transients in the cartesian space is obtained. Experimental results for a laboratory prototype prove the effectiveness of the proposed method.
\end{abstract}

Keywords: Mobile robots, nonholonomic systems, feedback linearization, tracking, stabilization.

\section{INTRODUCTION}

The control problem for robotic systems with nonholonomic constraints, such as wheeled mobile robots, free-floating space manipulators or multifingered robot hands, raises a particularly challenging theoretical issue. For these systems, feedback stabilization at a given equilibrium point (also called set-point regulation) turns out to be a difficult problem, since the tangent linearization of the system is uncontrollable and, in addition, smooth time-invariant stabilization is not possible (Brockett, 1983).

Set-point regulation has been tackled resorting to innovative control strategies, i.e., discontinuous (Canudas de Wit and Sørdalen, 1992), timevarying (Samson, 1995) and mixed (Sørdalen and Egeland, 1995) feedback laws; see (De Luca et al., 1998) for a complete bibliography. While these approaches overcome in principle the aforementioned difficulties, it should be acknowledged that their application brings along at least two practical drawbacks:
- System trajectories are almost invariably oscillatory and erratic, resulting in unpredictable motions. This may be a severe limitation, particularly for mobile robots in environments with obstacles.

- Control laws for set-point regulation are completely different from those used for trajectory tracking, which may be instead obtained by smooth feedback. Thus, the control module must implement different operation modes for each task, with additional complexity in the software architecture.

Our objective in this paper is to design a control scheme which is effective (in the sense that generates fast, predictable transients) for both trajectory tracking and set-point regulation tasks. For fully actuated robotic system without nonholonomic constraints, this goal can be achieved by the celebrated computed torque method, i.e., exact linearization via static feedback. For nonholonomic systems, we propose to adopt an extension of the same approach, namely exact linearization via dynamic feedback. This tool has 
been already used by d'Andrea-Novel et al. (1992) and by De Luca and Di Benedetto (1993) for trajectory tracking. Here, we show that it can be successfully exploited also for solving the set-point regulation problem. For illustration, the design procedure is carried out in full detail on a wheeled mobile robot with the kinematics of a unicycle. Experimental results are presented for the mobile robot SuperMARIO.

\section{CONTROL VIA DYNAMIC FEEDBACK LINEARIZATION}

Consider a nonlinear system

$$
\dot{q}=f(q)+g(q) w
$$

where $q$ is the $n$-dimensional state and $w$ is the $m$ dimensional input. For driftless robotic systems, typically arising with first-order kinematic models, it is $f(q) \equiv 0$ and the state is given by the generalized coordinates.

The dynamic feedback linearization problem consists in finding, if possible, a dynamic state feedback compensator of the form

$$
\begin{aligned}
\dot{\xi} & =a(q, \xi)+b(q, \xi) u \\
w & =c(q, \xi)+d(q, \xi) u,
\end{aligned}
$$

with $\nu$-dimensional state $\xi$ and $m$-dimensional new input $u$, such that the closed-loop system $(1-2)$ is equivalent, under a state transformation $z=T(q, \xi)$, to a linear controllable system.

Only sufficient or necessary conditions exist for the solution of the dynamic feedback linearization problem. Constructive algorithms, which are essentially based on input-output decoupling, can be found in (Isidori, 1995). For these, an $m$ dimensional system output $\eta=h(q)$ needs to be defined, to which a desired behavior can be assigned (e.g., track a given trajectory). One proceeds by successively differentiating the output until the input appears in a nonsingular way. At some stage, the addition of integrators on a subset of the input channels may be necessary in order to avoid subsequent differentiation of the original inputs. This dynamic extension builds up the state $\xi$ of the dynamic compensator (2). The algorithm terminates after a finite number of differentiations whenever the system is invertible from the chosen output. If the sum of the output differentiation orders equals the dimension $n+\nu$ of the extended state space, full input-state-output linearization is also obtained. The closed-loop system is then equivalent to a set of decoupled input-output chains of integrators from $u_{i}$ to $\eta_{i}(i=1, \ldots, m)$.

For first-order $n$-dimensional kinematic models of nonholonomic robots, the following facts have been established (De Luca et al., 1998):
- It is never possible to achieve exact linearization via static state feedback.

- A robot with $m=2$ inputs admitting a chained form representation (Sørdalen and Egeland, 1995) can always be exactly linearized via a dynamic feedback of dimension $\nu=n-2$. The resulting system consists of two decoupled chains of $n-1$ integrators.

\subsection{Application to the unicycle}

Let $q=(x, y, \vartheta)$ be the generalized coordinates of a unicycle, where $(x, y)$ is the cartesian position of the unicycle in a world frame and $\vartheta$ is its orientation with respect to the $x$ axis. The kinematic model of the system is

$$
\begin{aligned}
& \dot{x}=v \cos \vartheta \\
& \dot{y}=v \sin \vartheta \\
& \dot{\vartheta}=\omega,
\end{aligned}
$$

where $v$ and $\omega$ are respectively the driving and steering velocity inputs $(n=3$ and $m=2)$.

By taking $\eta=(x, y)$ as the system output, the dynamic linearization algorithm yields a controller of the form (De Luca et al., 1998)

$$
\begin{aligned}
& \dot{\xi}=u_{1} \cos \vartheta+u_{2} \sin \vartheta \\
& v=\xi \\
& \omega=\frac{-u_{1} \sin \vartheta+u_{2} \cos \vartheta}{\xi}
\end{aligned}
$$

being $\xi$ the state of the one-dimensional dynamic compensator $(\nu=1)$. In the new coordinates

$$
\begin{aligned}
& z_{1}=x \\
& z_{2}=y \\
& z_{3}=\dot{x}=\xi \cos \vartheta \\
& z_{4}=\dot{y}=\xi \sin \vartheta
\end{aligned}
$$

the extended system is fully described by two chains of two input-output integrators:

$$
\begin{aligned}
& \ddot{z}_{1}=u_{1} \\
& \ddot{z}_{2}=u_{2} .
\end{aligned}
$$

The linearizing control (4-6) law has a singularity at $\xi=v=0$, i.e., when the unicycle is not rolling. The occurrence of such singularity in the dynamic extension process is structural for nonholonomic systems (De Luca and Di Benedetto, 1993). This difficulty must be obviously taken into account when designing control laws for the equivalent linear model (11-12). 


\subsection{Trajectory tracking}

Assume the robot is to follow a smooth desired output trajectory $\left(x_{d}(t), y_{d}(t)\right)$ which is persistent, i.e., such that the square of the nominal control input $v_{d}^{2}=\dot{x}_{d}^{2}+\dot{y}_{d}^{2}$ along the trajectory never goes to zero.

On the linear and decoupled system (11-12), it is straightforward to design a globally exponentially stabilizing feedback to the desired trajectory (with linear cartesian transients) as

$$
\begin{aligned}
& u_{1}=\ddot{x}_{d}+k_{p 1}\left(x_{d}-x\right)+k_{d 1}\left(\dot{x}_{d}-\dot{x}\right) \\
& u_{2}=\ddot{y}_{d}+k_{p 2}\left(y_{d}-y\right)+k_{d 2}\left(\dot{y}_{d}-\dot{y}\right),
\end{aligned}
$$

with $k_{p i}>0, k_{d i}>0$, for $i=1,2$.

Even for smooth persistent trajectories, problems may arise if the actual command $v=\xi$ crosses zero during an initial transient. However, this situation can be avoided by suitably choosing the initial state $\xi_{0}$ of the dynamic compensator. For example, a simple way to keep the actual commands bounded is to reset the state $\xi$ whenever its value falls below a given threshold. This strategy results in an input command $v$ with isolated discontinuities with respect to time.

\subsection{Set-point regulation}

It is now shown that dynamic feedback linearization provides a solution also for the set-point regulation problem. Assume w.l.o.g. that the desired configuration $q^{d}$ is the origin of the configuration space $\mathcal{Q}=\mathbb{R}^{2} \times S O(1)$. We denote by

$$
\begin{aligned}
\mathcal{Q}^{*}=\{q \in \mathcal{Q}: & (x=0, \cos \vartheta \geq 0) \mathrm{OR} \\
& (y=0, \cos \vartheta=-1)\}
\end{aligned}
$$

a subset of $\mathcal{Q}$ which will require special attention. The remaining part $\mathcal{Q} / \mathcal{Q}^{*}$ of the configuration space can be partitioned in two regions:

$$
\begin{aligned}
& \mathcal{Q}^{r}=\left\{q \in \mathcal{Q} / \mathcal{Q}^{*}: x \geq 0\right\} \\
& \mathcal{Q}^{l}=\left\{q \in \mathcal{Q} / \mathcal{Q}^{*}: x<0\right\}
\end{aligned}
$$

Proposition 1. Consider the unicycle system (3) under the dynamic controller (4-6). A PD control law on the cartesian error

$$
\begin{aligned}
& u_{1}=-k_{p_{1}} x-k_{d 1} \dot{x} \\
& u_{2}=-k_{p 2} y-k_{d 2} \dot{y}
\end{aligned}
$$

yields exponential convergence to the origin from any initial configuration $q_{0}=\left(x_{0}, y_{0}, \vartheta_{0}\right) \in \mathcal{Q} / \mathcal{Q}^{*}$, if the following assumptions hold:
A1. The control gains $k_{p i}>0, k_{d i}>0(i=1,2)$ satisfy the conditions

$$
\begin{gathered}
k_{d 1}^{2}-4 k_{p 1}=k_{d 2}^{2}-4 k_{p 2}>0 \\
k_{d 2}-k_{d 1}>2 \sqrt{k_{d 2}^{2}-4 k_{p 2}} .
\end{gathered}
$$

A2. The initial state of the dynamic compensator is chosen as

$$
\begin{array}{ll}
\xi_{0}<0 \text { (backward motion) } & \text { if } q_{0} \in \mathcal{Q}^{r} \\
\xi_{0}>0 \text { (forward motion) } & \text { if } q_{0} \in \mathcal{Q}^{l},
\end{array}
$$

but its value is otherwise arbitrary, except for the additional condition

$$
\xi_{0} \neq 2 \frac{k_{p 1} x_{0} \sin \vartheta_{0}-k_{p 2} y_{0} \cos \vartheta_{0}}{k_{d 2}-k_{d 1}} .
$$

Proof. Use of control (13-14) in eqs. (11-12) imposes that the cartesian coordinates $x$ and $y$ converge to zero exponentially, provided that the original control inputs $v$ and $\omega$, given by eqs. (5) and (6), remain bounded. To show this, we must prove that (i) $\xi$ does not go to zero in finite time, and (ii) $\omega$ tends to zero for $t \rightarrow \infty$, in spite of its denominator $\xi$ vanishing.

(i) Since $\xi^{2}=z_{3}^{2}+z_{4}^{2}$ from eqs. (9-10), one has $\xi(\bar{t})=0$ iff $z_{3}(\bar{t})=z_{4}(\bar{t})=0$. Integrating the closed-loop system (11-12) under controls (13-14) from $t_{0}=0$, we have

$$
\begin{aligned}
& z_{3}(t)=a_{31} e^{\lambda_{11} t}+a_{32} e^{\lambda_{12} t} \\
& z_{4}(t)=a_{41} e^{\lambda_{21} t}+a_{42} e^{\lambda_{22} t},
\end{aligned}
$$

where coefficients $a_{k j}$ and eigenvalues $\lambda_{i j}$ are easily obtained as functions of initial state and PD gains. From these expressions and condition (15), it is possible to show that a finite $\bar{t}>0$ such that $\xi(\bar{t})=0$ exists iff

$$
\frac{\xi_{0} \cos \vartheta_{0}-x_{0} \lambda_{11}}{\xi_{0} \cos \vartheta_{0}-x_{0} \lambda_{12}}=\alpha \frac{\xi_{0} \sin \vartheta_{0}-y_{0} \lambda_{21}}{\xi_{0} \sin \vartheta_{0}-y_{0} \lambda_{22}}
$$

with $\alpha=\lambda_{11} \lambda_{22} / \lambda_{12} \lambda_{21}$. From this, a quadratic equation in $\xi_{0}$ is derived which has the single nonzero root

$\xi_{0}=\frac{\lambda_{11} x_{0} \sin \vartheta_{0}+\lambda_{22} y_{0} \cos \vartheta_{0}-\alpha\left(\lambda_{12} x_{0} \sin \vartheta_{0}+\lambda_{21} y_{0} \cos \vartheta_{0}\right)}{(1-\alpha) \sin \vartheta_{0} \cos \vartheta_{0}}$.

Once rewritten in terms of the $\mathrm{PD}$ gains, this expression leads to condition (17).

(ii) First of all, it is straightforward to prove that assumption A1 implies that the eigenvalues are real and ordered as $\lambda_{11}<\lambda_{12}<\lambda_{21}<\lambda_{22}<0$. Rewriting eq. (6) as

$$
\omega=\frac{-z_{4} u_{1}+z_{3} u_{2}}{\xi^{2}},
$$

and using eqs. (18-19) and (13-14), the numerator of $\omega$ takes the form 


$$
\begin{aligned}
-z_{4} u_{1}+z_{3} u_{2}= & \gamma_{1} e^{\left(\lambda_{11}+\lambda_{21}\right) t}+\gamma_{2} e^{\left(\lambda_{12}+\lambda_{21}\right) t}+ \\
& \gamma_{3} e^{\left(\lambda_{11}+\lambda_{22}\right) t}+\gamma_{4} e^{\left(\lambda_{12}+\lambda_{22}\right) t}
\end{aligned}
$$

with $\gamma_{i} \in \mathbb{R}$. Its asymptotic rate of convergence is certainly larger than $2\left|\lambda_{22}\right|$ due to the eigenvalue ordering. As for the denominator, squaring and adding eqs. (18) and (19) gives

$$
\begin{gathered}
\xi^{2}=\eta_{1} e^{2 \lambda_{11} t}+\eta_{2} e^{\left(\lambda_{11}+\lambda_{12}\right) t}+\eta_{3} e^{2 \lambda_{12} t}+ \\
\eta_{4} e^{2 \lambda_{21} t}+\eta_{5} e^{\left(\lambda_{21}+\lambda_{22}\right) t}+\eta_{6} e^{2 \lambda_{22} t}
\end{gathered}
$$

with $\eta_{i} \in \mathbb{R}$. Since the asymptotic rate of convergence of this quantity is exactly $2\left|\lambda_{22}\right|$, we conclude that $\omega$ tends to zero as $t \rightarrow \infty$.

To finish the proof, it is necessary to show that also the orientation $\vartheta$ converges to zero. This is easily understood from the following facts:

- The unicycle reaches the origin with a horizontal tangent $(\vartheta=0$ or $\pi$ ), because $y$ approaches zero faster than $x$ in view of the eigenvalue ordering.

- Motion inversions do not occur since $v=\xi$ never crosses zero, as shown in the first part of the proof.

- The trajectory is confined to the region (either $\mathcal{Q}_{r}$ or $\mathcal{Q}_{l}$ ) from which the unicycle starts. In fact, $x$ and $y$ never change sign because the eigenvalues are real and thanks to the choice of sign for $\xi_{0}$ in assumption A2.

Finally, also the convergence of $\vartheta$ to zero is exponential. Indeed, since $\omega$ goes exponentially to zero, the same is true for its integral $\vartheta$.

A few remarks are in order.

- As the cartesian position transients are linear, the unicycle trajectories obtained with the proposed controller are highly predictable and can be easily shaped by choosing the PD control gains.

- The equality part of condition (15) in Proposition 1 is by no means necessary, but is used only for deriving a closed form for the forbidden initialization (17) of the dynamic compensator.

- In view of the discontinuity at the origin of the linearizing controller with respect to the state $(x, y, \vartheta, \xi)$ of the extended system, as well as of the fact that the initial configuration should belong to $\mathcal{Q} / \mathcal{Q}^{*}$, the proposed feedback controller does not yield Lyapunov stability in a strict sense, but simply exponential convergence.

If the initial configuration $q_{0}$ belongs to $\mathcal{Q}^{*}$, Prop. 1 cannot be applied. In fact, the PD control (13-14) would bring the unicycle to the origin with the wrong orientation $\left(\vartheta=\pi\right.$ if $\cos \vartheta_{0}>0$, $\vartheta= \pm \pi / 2$ if $\cos \vartheta_{0}=0, \vartheta=-\pi$ if $\cos \vartheta_{0}=-1$ ). In such situation, it is necessary to reset the com-

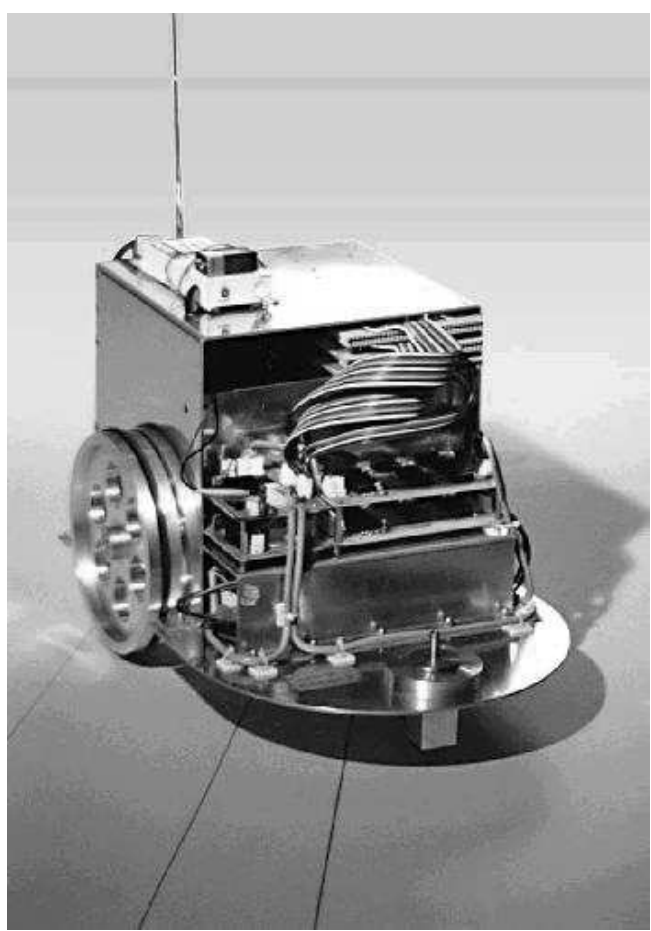

Fig. 1. The wheeled mobile robot SuperMARIO pensator state at some time $t_{v}>0$, so as to invert the motion at a configuration $q \in \mathcal{Q} / \mathcal{Q}^{*}$. A simple way to obtain this is to introduce a via point in the regulation procedure, as illustrated in the next section by a parallel parking experiment.

\section{EXPERIMENTAL RESULTS}

Experimental validation of the proposed dynamic feedback linearization strategy has been performed on the mobile robot SuperMARIO available at the Robotics Laboratory of our department and shown in Fig. 1. The chassis is made of aluminum and measures $46 \times 32 \mathrm{~cm}$, while the total weight of the robot is about $8 \mathrm{~kg}$. SuperMARIO is equipped with two actuated wheels (of radius of $9.93 \mathrm{~cm}$ ) on the same axle (of length $29 \mathrm{~cm}$ ), independently driven by two DC servomotors, and a castor wheel. The kinematic model of the vehicle is equivalent to the unicycle model (3). On-board electronics includes two encoders mounted on the driving wheels, two PID low-level wheel velocity loops, and two serial cards allowing communication with a remote control computer (a $300 \mathrm{MHz}$ Pentium II) at the maximum speed of $4800 \mathrm{bit} / \mathrm{s}$ via a radio-link.

The sampling period used in the experiments is 50 ms. Custom algorithms have been developed to reduce the effect of quantization errors and communication delays in the reconstruction of the state from the odometric information provided by the encoders. A calibration procedure has also been performed to estimate the actual wheel radii and axle length. The system shows however the 
typical limitations of a low-cost prototype, and is prone to a variety of errors due to mechanical friction, gear backlash, wheel slippage and other non-idealities.

Both trajectory tracking and set-point regulation tasks have been executed, but we report only on the latter for which we have chosen the gains $k_{p 1}=2, k_{d 1}=3, k_{p 2}=12$, and $k_{d 2}=7$, thus satisfying assumption A1.

The first experiment is a typical forward parking: the initial configuration is $(-1,1,0)$ (m,m,rad) while the goal is the origin. Figure 2 shows the cartesian motion of the robot, with the expected linear transient. To prevent wheel slippage, the velocity inputs $v$ and $\omega$ are not allowed to exceed in modulus the maximum values $v_{\max }=0.5(\mathrm{~m} / \mathrm{s})$ and $\omega_{\max }=0.3(\mathrm{rad} / \mathrm{s})$. The effect of this software saturation can be seen in Figs. 3-4.

A parallel parking maneuver is the assigned task in the second experiment. The robot starts at $(0,1.2,0)(\mathrm{m}, \mathrm{m}, \mathrm{rad})$ and the goal is again the origin. As mentioned at the end of the previous section, the reset of the dynamic compensator $\xi$ is required in this situation. In fact, the PD control law (13-14) as such would bring the unicycle to the origin with the wrong orientation $\vartheta=\pi$. The problem is easily solved with the introduction of the via point $q_{v}=(-0.6,-1,0)$ to which the robot converges in the first phase of the regulation task, under the action of a PD control designed with the $q_{v}$ as the (intermediate) goal. The state $\xi$ of the dynamic compensator is reset as soon as the position error with respect to the via point falls below a given threshold. At this time, the control law (13-14) kicks in and the robot converges exponentially to the origin with the correct orientation. Both the via point selection and the reset procedure have been automatized through a simple preprocessing step.

Figure 5 shows the cartesian motion of the robot. To account for actuator dynamics, a simple firstorder linear filter has been introduced so as to avoid the discontinuity in the driving velocity generated by the reset procedure. A similar filtering plus saturation procedure has been used to neutralize the effect of the singularity in the steering velocity due to the zero crossing of the filtered driving velocity. Figures $6-7$ show the profiles of the obtained driving and steering velocity, respectively. Note also that, as a consequence of the filtering, the actual velocity inputs are zero at $t_{0}=0$ even if we have chosen $\left|\xi_{0}\right|=v_{\max }$.

\section{CONCLUSIONS}

We have considered the feedback control problem for a nonholonomic wheeled vehicle with the kine- matics of a unicycle. It has been shown that exact linearization via dynamic feedback can be used to solve not only trajectory tracking but also setpoint regulation problems.

The main advantages of the presented approach are that (i) linear cartesian transients with exponential rate of convergence are obtained, and (ii) the same, very simple control law works in both cases. The effectiveness of our control strategy has been shown by experimental results for a laboratory prototype.

Among the directions of further research, we cite the extension of the proposed regulation technique to other nonholonomic vehicles such as the carlike robot, with or without trailers. In fact, as shown in (De Luca et al., 1998), dynamic feedback linearization is always possible for these systems.

\section{ACKNOWLEDGEMENTS}

This work was supported by MURST within the RAMSETE Project.

\section{REFERENCES}

Brockett, R. W. (1983). Asymptotic stability and feedback stabilization. In: Differential Geometric Control Theory (R. W. Brockett, R. S. Millman and H. J. Sussmann, Eds.). Pp. 181191. Birkhäuser.

Canudas de Wit, C. and O.J. Sørdalen (1992). Exponential stabilization of mobile robots with nonholonomic constraints. IEEE Trans. on Automatic Control, 37(11), 1791-1797.

De Luca, A. and M. D. Di Benedetto (1993). Control of nonholonomic systems via dynamic compensation. Kybernetika, 29(6), 593-608.

De Luca, A., G. Oriolo and C. Samson (1998). Feedback control of a nonholonomic car-like robot. In: Robot Motion Planning and Control (J.-P. Laumond, Ed.). Pp. 171-253. SpringerVerlag.

Isidori, A. (1995). Nonlinear Control Systems. Springer-Verlag.

Samson, C. (1995). Control of chained systems. application to path following and time-varying point-stabilization of mobile robots. IEEE Trans. on Automatic Control, 40(1), 64-77.

Sørdalen, O. J. and O. Egeland (1995). Exponential stabilization of nonholonomic chained systems. IEEE Trans. on Automatic Control, 40(1), 35-49. 


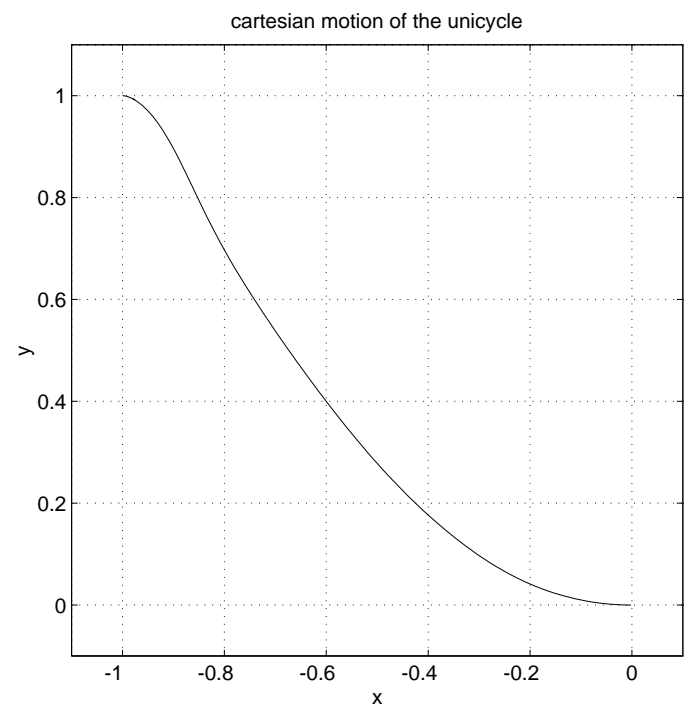

Fig. 2. First experiment: Cartesian motion of the unicycle

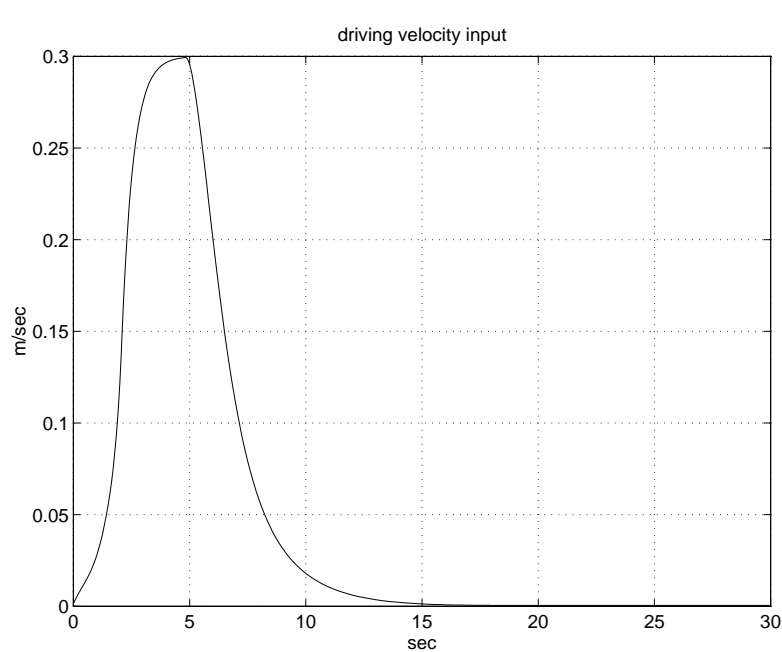

Fig. 3. First experiment: Driving velocity $v$

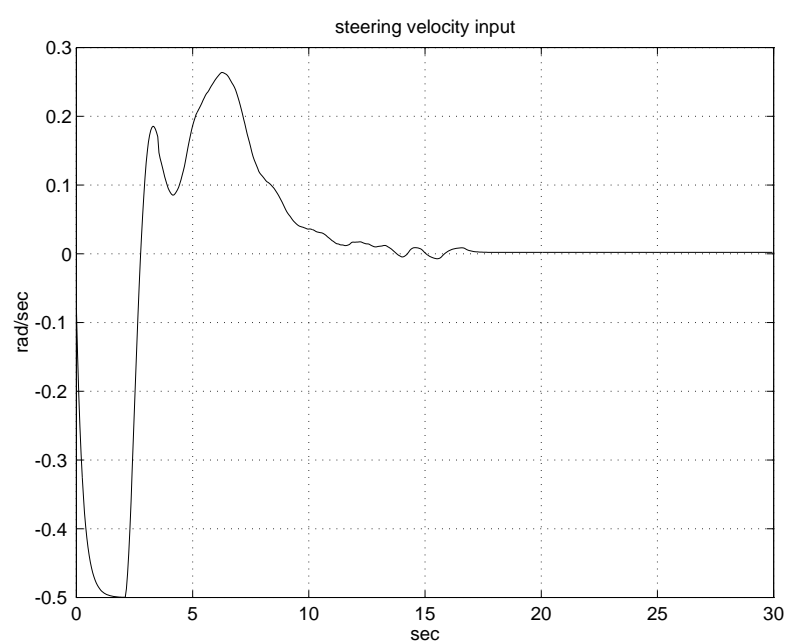

Fig. 4. First experiment: Steering velocity $\omega$

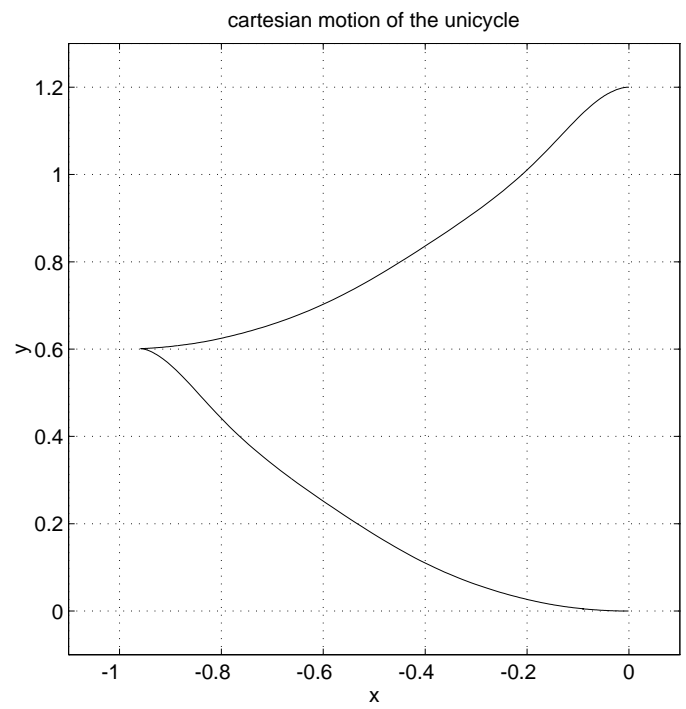

Fig. 5. Second experiment: Cartesian motion of the unicycle

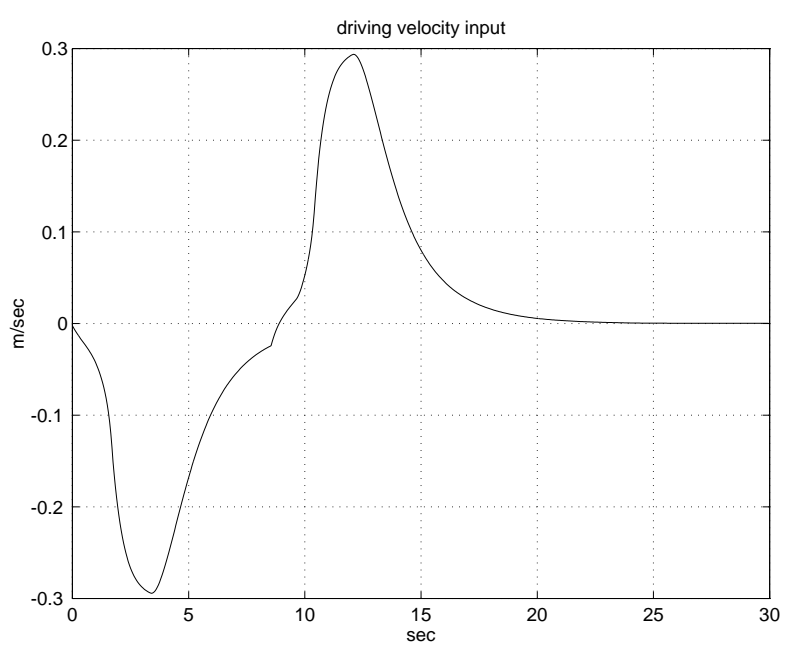

Fig. 6. Second experiment: Driving velocity $v$

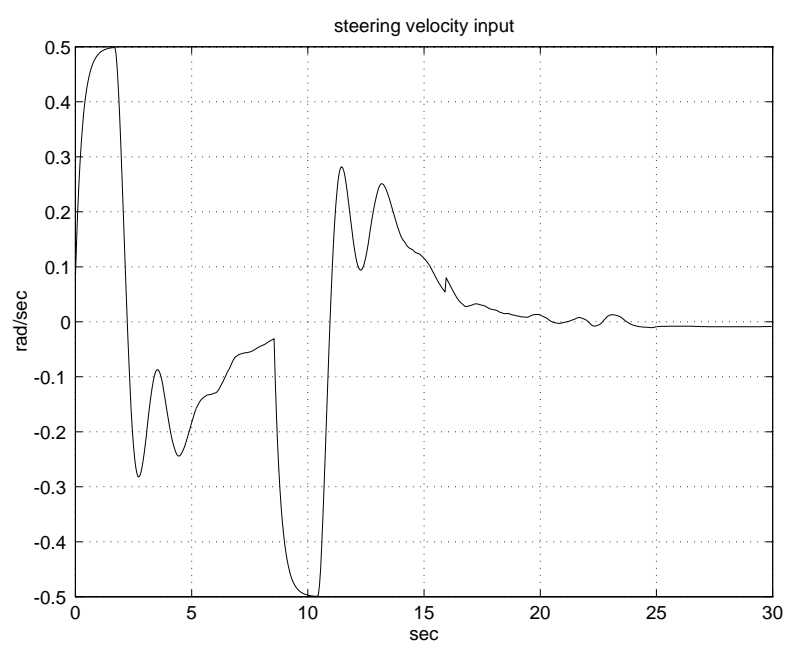

Fig. 7. Second experiment: Steering velocity $\omega$ 